\title{
Medición del potencial de explotación de energía fotovoltaica y eólica en Tamaulipas, región Altamira
}

\section{Measurement of the potential of the exploitation of photovoltaic and wind energy in Tamaulipas, region Altamira}

\author{
CASTILLO-MARTINEZ, Oscar*†, BORTONI-ANZURES, Liborio Jesus y RODRIGUEZ-CHAVEZ, \\ Ulises
}

Universidad Politecnica de Victoria

ID $1^{\text {er }}$ Autor: Oscar, Castillo-Martinez / ORC ID: 0000-0003-4247-8910, CVU CONACYT ID: 982880

ID $1^{\mathrm{er}}$ Coautor: Liborio Jesus, Bortoni-Anzures / ORC ID: 0000-0003-2025-4369, CVU CONACYT ID: 92743

ID 2 ${ }^{\text {do }}$ Coautor: Ulises, Rodriguez-Chavez / ORC ID: 0000-0001-5203-5262, CVU CONACYT ID: 983900

DOI: $10.35429 /$ JSI.2019.12.3.1.3

Recibido 02 de Agosto, 2019; Aceptado 30 de Noviembre, 2019

\section{Resumen}

Actualmente en México se busca generar energía eléctrica por medio de recursos renovables o limpios de acuerdo al marco normativo y legal, en este proyecto se construirá una estación meteorológica portátil y se realizarán mediciones del viento, dirección de viento, medición de irradiación y temperatura, estos datos se estarán adquiriendo y almacenando en tiempo real atravez de un circuito electrónico llamado data logger basado en microprocesadores y memorias. Estos datos serán procesados matemáticamente y graficados para realizar un estudio de la medición del potencial de explotación de energía fotovoltaica y eólica en Tamaulipas, región Altamira, en base a los datos de las variables ambientales medidas y recolectadas durante cierto tiempo. Esta investigación tiene por objetivo contribuir con un estudio que determine la viabilidad de la generación de energía eléctrica por medio de recursos renovables fotovoltaicos y eólicos en esta región en específico y promover el desarrollo sustentable de la industria eléctrica. Objetivos: Construir una estación meteorológica portátil y realizar un estudio de la medición del potencial de explotación de energía fotovoltaica y eólica en Tamaulipas, región Altamira, en base a los datos de las variables ambientales medidas y recolectadas durante cierto tiempo, para determinar la viabilidad de la generación eléctrica mediante recurso eólico y solar en esta región. Metodología: Para poder realizar la evaluación de la medición del potencial de energía fotovoltaica y eólico en Tamaulipas, región Altamira, se fabricará un módulo de estación meteorológica portátil personalizado, para realizar mediciones de la velocidad del viento, mediciones de la dirección del viento, mediciones de temperatura, y mediciones de irradiación, en el sitio de interés. Además se instalara un sistema para registrar todas las variables descritas en una memoria micro SD y un sistema de comunicación a través de un módulo bluetooth. Esta estación meteorológica portátil se instalará, en la parte superior del edificio de biblioteca de la Universidad Politécnica de Altamira. La ubicación geográfica exacta es mostrada por medio de Google Maps con una Latitud 22.462239 y longitud: -97.970186 (Google, 2018). Contribución: Proporcionar información de las variables meteorológicas de nuestro entorno, añadiéndole un panel solar que demuestre su viabilidad. Implementar un interfaz, que permita el monitoreo constante y las diferentes mediciones realizadas en la estación meteorológica, mediante sensores y microntroladores.

Energía, Fotovoltaico, Eólico

\author{
Abstract
}

Now a day, Mexico is looking the way to generate electricity by using renewable, clean and ecofriendly sources according the actual legal framework. This project is a meteorological portable station which will record either direction and speed of wind, light irradiation and environment air temperature in a data logger unit conformed by a microprocessor and a memory. The data recorded in the device will be mathematically analyzed and processed in a delimited period of time and plotted on different type of graphs or documents for to support feasibilities studies which could identify the power potential of photovoltaic energy and wind energy in the city of Altamira Tamaulipas. The main goal of this project is contribute with actual studies which will determine the feasibility of energy generation by using clean energy sources in the south part of our state promoting the development of energy power industry, mainly in the city of Altamira. Objectives: To build a portable meteorological station and to carry out a study of the measurement of the photovoltaic and wind energy exploitation potential in Tamaulipas, Altamira region, based on the data of the environmental variables measured and collected during a certain time, to determine the viability of generation electricity through wind and solar resource in this region. Methodology: In order to carry out the evaluation of the photovoltaic and wind energy potential measurement in Tamaulipas, Altamira region, a customized portable weather station module will be manufactured, to perform measurements of wind speed, wind direction measurements, temperature measurements, and irradiation measurements, at the site of interest. In addition, a system will be installed to register all the variables described in a micro SD memory and a communication system through a bluetooth module. This portable weather station will be installed, at the top of the library building of the Polytechnic University of Altamira. The exact geographical location is shown by means of Google Maps with a Latitude 22.462239 and longitude: -97.970186 (Google, 2018). Contribution: Provide information on the meteorological variables of our environment, adding a solar panel that demonstrates its viability. Implement an interface that allows constant monitoring and the different measurements made at the meteorological station, using sensors and microntrollers.

Energy, Photovoltaic, Wind Power

Citación: CASTILLO-MARTINEZ, Oscar, BORTONI-ANZURES, Liborio Jesus y RODRIGUEZ-CHAVEZ, Ulises. Medición del potencial de explotación de energía fotovoltaica y eólica en Tamaulipas, región Altamira. Revista de Innovación Sistemática. 2019. 3-12:1-3

*Correspondencia al Autor (Correo electrónico: 1819018@upv.edu.mx)

$\dagger$ Investigador contribuyendo como primer Autor. 


\section{Introducción}

Una de las problemáticas en la actualidad es la contaminación a base de combustibles fósiles y sus derivados, los cuales generan el efecto invernadero y deterioro de la capa de ozono, ocasionando el calentamiento global.

Una de los sectores industriales que más contamina es el energético, por lo actualmente en México existen reformas que tienen por finalidad promover el desarrollo sustentable de la industria eléctrica y garantizar su operación continua, eficiente y segura en beneficio de los usuarios, así como el cumplimiento de las obligaciones de servicio público y universal, de Energías Limpias y de reducción de emisiones contaminantes.

\section{frecuencia relatiiva vs vel.prom.}

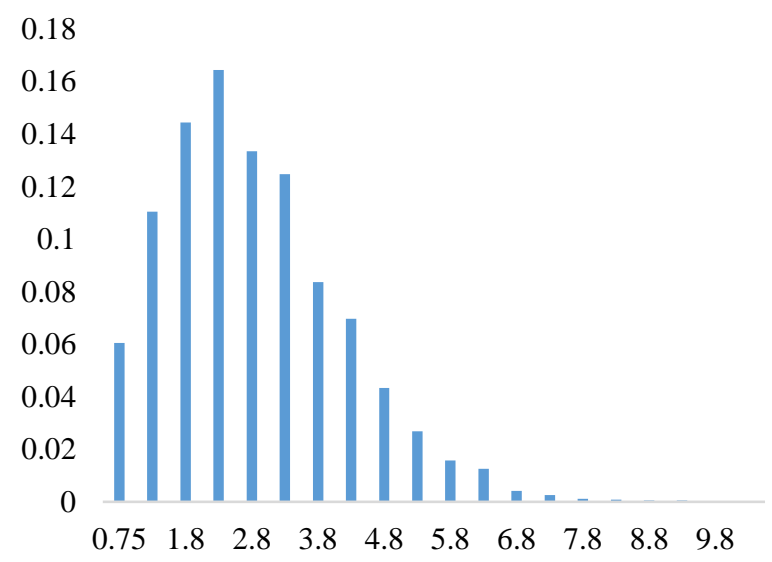

Gráfico 1 comparativa de la velocidad promedio Fuente: Elaboración Propia

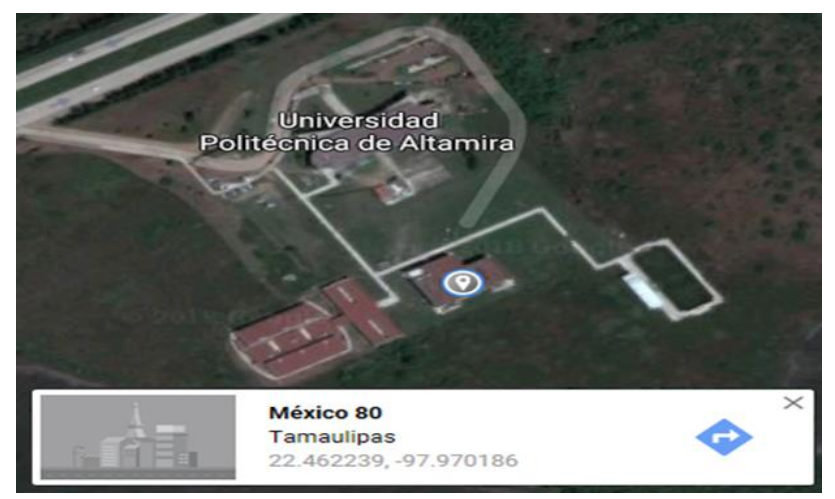

Figura 1 Ubicación de estación meteorológica portátil Fuente:

https://www.google.com.mx/maps/place/UPALT(Universi dad+Politécnica+de+Altamira)/@22.4632086,-

97.9709646,256m/data $=$ !3m1!1e3!4m5!3m4!1 s0x867808 6959821de3:0xa4999160150f292!8m2!3d22.4634032!4d $-97.97086$ $3.57 \mathrm{~m} / \mathrm{s}$.

La velocidad promedio de este día fue de

\begin{tabular}{|c|c|c|c|}
\hline Temp. Prom & 29.94 & $3.57 \mathrm{~m} / \mathrm{s}$ & V.Prom \\
\hline Altura sobre nivel del mar & 6.09 Metros & & \\
\hline \multicolumn{4}{|l|}{ ejemplo $1427 / 23554=0.6049$} \\
\hline Frecuencia Relativa (fr) fr/N & $\begin{array}{l}\text { Frecuencia } \\
\text { Relativa } \\
\text { Acumulada } \\
\text { (fa) }\end{array}$ & $\begin{array}{l}\text { Frecuencia } \\
\text { Acumulada }\end{array}$ & $\begin{array}{c}\text { Frecuencia } \\
\text { Relativa } \\
\text { Porcentual } \\
100 * \mathrm{fr}\end{array}$ \\
\hline 0.060499278 & 0.060499278 & 1425 & 6.049927825 \\
\hline 0.110427104 & 0.170926382 & 4026 & 11.04271037 \\
\hline 0.144349155 & 0.315275537 & 7426 & 14.43491551 \\
\hline 0.164345759 & 0.479621296 & 11297 & 16.43457587 \\
\hline 0.133395602 & 0.613016897 & 14439 & 13.33956016 \\
\hline 0.124692197 & 0.737709094 & 17376 & 12.46921967 \\
\hline 0.083595143 & 0.821304237 & 19345 & 8.359514308 \\
\hline 0.069754606 & 0.891058844 & 20988 & 6.975460644 \\
\hline 0.043304747 & 0.93436359 & 22008 & 4.330474654 \\
\hline 0.026831961 & 0.961195551 & 22640 & 2.68319606 \\
\hline 0.015793496 & 0.976989046 & 23012 & 1.57934958 \\
\hline 0.012651779 & 0.989640825 & 23310 & 1.265177889 \\
\hline 0.004203108 & 0.993843933 & 23409 & 0.420310775 \\
\hline 0.002632249 & 0.996476182 & 23471 & 0.26322493 \\
\hline 0.001231213 & 0.997707396 & 23500 & 0.123121338 \\
\hline 0.000764201 & 0.998471597 & 23518 & 0.076420141 \\
\hline 0.000551923 & 0.99902352 & 23531 & 0.055192324 \\
\hline 0.000509468 & 0.999532988 & 23543 & 0.050946761 \\
\hline 0.000254734 & 0.999787722 & 23549 & 0.02547338 \\
\hline 0.000212278 & 1 & 23554 & 0.021227817 \\
\hline
\end{tabular}

Tabla 1 Tabla de cálculo de velocidades promedio de un día

Fuente: Elaboración Propia

\section{Metodología a desarrollar}

Para poder realizar la evaluación de la medición del potencial de energía fotovoltaica y eólico en Tamaulipas, región Altamira, se fabricara un módulo de estación meteorológica portátil personalizado, para realizar mediciones de la velocidad del viento, mediciones de la dirección del viento, mediciones de temperatura, y mediciones de irradiación, en el sitio de interés. Además se instalara un sistema para registrar todas las variables descritas en una memoria micro SD y un sistema de comunicación a través de un módulo bluetooth. Esta estación meteorológica portátil se instalara, en la parte superior de la Universidad Politécnica de Altamira. La ubicación geográfica exacta es mostrada por medio de Google Maps con una Latitud 22.462239 y longitud: -97.970186.

\section{Resultados}

Con este trabajo se presentaran las variables registradas de la estación meteorológica portátil obtenidas en los 12 meses del año 2019, durante este periodo de muestreo de cada 15 min por muestra, aproximadamente 96 muestras diarias, a una altura de $9 \mathrm{mts}$., se tiene estimado lograr un total de 35040 muestras. Después se eliminaran registros con valores de $0 \mathrm{~m} / \mathrm{s}$, debido a que no tiene un valor de energía cinética del viento.

CASTILLO-MARTINEZ, Oscar, BORTONI-ANZURES, Liborio Jesus y RODRIGUEZ-CHAVEZ, Ulises. Medición del potencial de explotación de energía fotovoltaica y eólica en Tamaulipas, región Altamira. Revista de Innovación Sistemática. 2019. 
Por tal motivo actualmente no se ha terminado el estudio y análisis para determinar si es factible el área para futuros emplazamientos.

\section{Agradecimiento}

Se agradece a la Universidad Politécnica de Altamira, por las facilidades para la instalación de la estación meteorológica en la azotea de uno de sus edificios y continuar con la recolección de datos para realizar este estudio.

\section{Conclusiones}

Hoy en día existen investigaciones relacionadas con esta investigación pero no en el sitio de nuestro interés, ya que en otras partes del mundo es de gran interés el tema para realizar futuros emplazamientos relacionados con las energías fotovoltaicas y eólicas.

\section{Referencias}

Hernández Escobedo Quetzalcóatl, febrero 2012, Evaluación del potencial eólico para la generación de energía eléctrica en el estado de Veracruz, México, Dyna, vol. 79, núm. 171. https://www.redalyc.org/pdf/496/49623207028. pdf

Rodríguez Aya Ángel Alejandro, (2018). Análisis del potencial energético solar basado en mediciones in situ en el municipio de Acacías Meta.

https://www.knepublishing.com/index.php/KnE -Engineering/article/view/1472

Vergara Barrios Pedro Pablo, 2014, Evaluación del potencial solar y eólico del campus central de la universidad industrial de Santander y la ciudad de Bucaramanga, Colombia. UIS Ingeniería, https://revistamedicasuis.uis.edu.co/index.php/r evistauisingenierias/article/download/49-

$57 / 4729$ 